RESEARCH REPORT

\section{Health-related beliefs and experiences of Gypsies and Travellers: a qualitative study}
Patrice Van Cleemput, Glenys Parry, Kate Thomas, Jean Peters, Cindy Cooper

政

This paper is freely available online under the BMJ Journals unlocked scheme, see http://jech.com/info/unlocked.dtl
See end of article for authors' affiliations ......................

Correspondence to: P V Cleemput, School of Health and Related Research, University of Sheffield, Regent Court, 30 Regent Street, Sheffield S1 4DA, UK; p.vancleemput@ sheffield.ac.uk

Accepted 16 September 2006

\begin{abstract}
Objective: To illuminate findings of the survey of the health status of Gypsies and Travellers by exploring their health-related beliefs and experiences.

Design: Qualitative study of a purposive subsample from in-depth interviews using framework analysis. Setting: The homes or alternative community settings of the participants in five geographically dispersed study locations in England.

Participants: 27 Gypsies and Travellers with an experience of ill health, purposively sampled from a larger population participating in an epidemiological survey of health status.

Results: The experience of poor health and daily encounters of ill health among extended family members were normalised and accepted. Four major themes emerged relating to health beliefs and the effect of lifestyle on health for these respondents: the travelling way; low expectations of health; self-reliance and staying in control; fatalism and fear of death. These themes dominated accounts of health experience and were relevant to the experience. These themes add richness to the health status data and inform our understanding.

Conclusions: Among Gypsies and Travellers, coherent cultural beliefs and attitudes underpin health-related behaviour, and health experiences must be understood in this context. In this group, ill health is seen as normal, an inevitable consequence of adverse social experiences, and is stoically and fatalistically accepted. The provision of effective healthcare and improvement of poor health in Gypsies and Travellers will require multi-agency awareness of these issues.
\end{abstract}

$\mathrm{W}$ orldwide there are more than 100 groups of Gypsies and Travellers who collectively form part of a wider Romani population, estimated at 12 million people. The Gypsies and Travellers in this study comprise English and Welsh Gypsies, Irish Travellers and Scottish Gypsy Travellers. English and Welsh Gypsies and Irish Travellers form an estimated population of 300000 in England and Wales. ${ }^{12}$ Nomadism and caravan (trailer) dwelling, although not defining them, are features of their cultural heritage rather than a lifestyle choice; many live in houses and others live on authorised or non-authorised residential caravan sites. $^{3}$ They are among the UK's most socially excluded groups $^{4}$ despite legal protection as a result of their ethnic minority status.

There are many previous studies of health meanings, values and practices among different cultural groups, and studies concerning beliefs and views about specific health problems. However, there seem to be little information concerning general views and perceptions of health and influences on the health of Gypsies and Travellers.

This was not a comparative or theoretically informed study, but a descriptive qualitative strand of an applied policy mixed methods study designed to estimate the current health status of Gypsies and Travellers. The key finding from the health status survey was the high burden of ill health among Gypsies and Travellers. ${ }^{5}$ We designed the qualitative study using a framework approach ${ }^{6}$ to put their health status into context by purposively selecting a sample to explore how ill health was experienced and understood by this minority group.

There are few previous qualitative studies on the health of Gypsies or Travellers in the UK and Ireland, ${ }^{7-9}$ and most report anecdotal data from practitioner accounts. ${ }^{10-15}$

\section{METHODS}

\section{Participants and setting}

The sampling frame comprised 269 Gypsies and Travellers participating in a parallel study of their health status conducted at five English locations: the survey sample was itself constructed to include quotas of men and women, Irish and British, across roadside, official, private and housed accommodation. Within this frame, anyone who reported a recent or current health problem and therefore had cause to access health services was eligible for inclusion in the qualitative study (ie, intensity sampling). Maximum variation purposive sampling $^{16}$ was also used across the study localities to ensure a representative final sample (table 1). A variety of health problems or unidentified symptoms were reported among the final sample (appendix A). Interviews were distributed over a 12-month period to capture any seasonal variation in mood and health states.

Research governance approval (including multicentre research ethics committee ethical review) was obtained with secondary approval from primary care trusts and local research ethics committees in each location where health visitors worked.

\section{Interviews}

Data were collected by individual in-depth semistructured interviews with participants in their own homes or at a venue of their choice. The interviews were carried out within 23 weeks of completion of the Health Survey Questionnaire. A topic guide was developed from five sources: personal practitioner experience; previous pilot study ${ }^{17}$; other published evidence; advice from the project advisory team, which included Gypsy Travellers; and initial pilot interviews. Nondirective interview questions allowed the exploration of 
Table 1 Final sampling grid

\begin{tabular}{|c|c|c|}
\hline Characteristics & $\begin{array}{l}\text { Number from } \\
\text { interviewed } \\
\text { sample }(n=27)\end{array}$ & $\begin{array}{l}\text { Number from } \\
\text { whole sample } \\
\text { ( } n=269 \text { ) }\end{array}$ \\
\hline \multicolumn{3}{|l|}{ Sex } \\
\hline Men & $7(25)$ & $96(36)$ \\
\hline Women & $20(74)$ & $173(64)$ \\
\hline \multicolumn{3}{|l|}{ Age group (years) } \\
\hline $16-25$ & $4(15)$ & $64(24)$ \\
\hline $26-45$ & $13(48)$ & $138(51)$ \\
\hline $46-65$ & $7(26)$ & $56(21)$ \\
\hline$>65$ & $3(11)$ & $11(4)$ \\
\hline \multicolumn{3}{|l|}{ Accommodation } \\
\hline Council site/private site & $16(59)$ & $114(42)$ \\
\hline Unauthorised roadside site & $2(7)$ & $75(28)$ \\
\hline Housed & $8(30)$ & $73(27)$ \\
\hline In temporary (homeless) & $1(4)$ & $7(3)$ \\
\hline \multicolumn{3}{|l|}{ Location (pilot) } \\
\hline Northampton & $2(7)$ & $5(2)$ \\
\hline Sheffield & $0(0)$ & $60(22)$ \\
\hline Bristol & $4(14)$ & $56(21)$ \\
\hline Norfolk & $3(11)$ & $66(25)$ \\
\hline London & $8(30)$ & $54(21)$ \\
\hline Leicester & $10(37)$ & $57(21)$ \\
\hline Attended school regularly as a child & $13(77)^{*}$ & $118(60)$ \\
\hline Have children $<16$ years & $21(88)^{*}$ & $229(85)$ \\
\hline \multicolumn{3}{|l|}{ Travelling patterns } \\
\hline All of the time & $0(0)^{*}$ & 71 (27) \\
\hline Part of the time & $5(21)^{*}$ & $70(26)$ \\
\hline Rarely & $19(79)^{*}$ & $125(47)$ \\
\hline
\end{tabular}

health experience, access and use of health services, approaches to preventive healthcare, health knowledge, effect of lifestyle on health and health beliefs. The interviews were long and detailed; this paper focuses on the last two topic areas and describes the four major emergent themes relating to them.

Interviews were conducted by the lead author (PVC); all but one were tape recorded.

\section{Analysis}

The software package Atlas $\mathrm{Ti}^{\mathrm{TM}}$ (developed by Thomas Muhr, Scientific Software Development, Berlin) was used for organisation and retrieval of data. A thematically organised understanding was developed using the framework method. ${ }^{18}$ This is designed for use in applied qualitative research to inform social policy, allowing for rigorous and transparent data management.

Analysis proceeded reiteratively in four stages: coding, thematic framework, descriptive accounts and interpretative analyses. Each stage was conducted by the first author (PVC) and peer reviewed by the second author (GP). The final version of each stage was also reviewed by the third author (KT).

\section{Respondent validation}

To test our interpretation of the data and to check for counterexamples, credibility, confidentiality and transferability, ${ }^{6}$ we presented the initial thematic findings to volunteer groups of Gypsies and Travellers in each study location. Volunteers attended by open invitation. To examine transferability of findings to the wider Gypsy and Traveller community, they were not selected by health status. A total of 55 attended these five forums. We asked them for their opinions from their own experience and also whether they felt these findings would be true for other groups of Gypsies and Travellers. No concerns were raised about confidentiality, and none of the emerging findings was challenged. Individual themes were confirmed and elaborated. They were recognised as meaningful and important to an understanding of Gypsies and Travellers in relation to health. Feedback from these groups suggested that the findings were transferable to the wider Gypsy and Traveller community.

\section{RESULTS}

To achieve the purposive sample of 27 interviews, 59 Gypsies and Travellers who originally consented were approached. The most common reasons for failed contacts were family illness or crisis, funeral attendances or the family having moved on since initial contact at the survey stage. It is therefore possible that the obtained sample is less mobile and less likely to be in crisis than the population as a whole. However, because the obtained sample varied as intended across the specified range of personal characteristics (table 1), it is less likely that transferability of findings would be affected by the number of failed contacts. The final sample included the last two of five pilot interviews.

Although the sample consisted of different ethnic Gypsy and Traveller groups from the UK and Ireland, participants expressed a strong sense of shared identity as "Gypsy Travellers". Although some Irish Travellers do move between Ireland and England, most are permanently resident as secondgeneration Irish Travellers. All those in this study were permanently resident in England. Four themes emerged, which were particularly relevant to their health experience.

\section{The Travelling Way}

Social and cultural issues permeated accounts of health and illness. Key among these were accommodation issues and issues associated with a travelling lifestyle. All participants chose to describe their current situation in relation to their ability to travel. Despite now living in a range of accommodation types, virtually all participants had experienced the "traditional" travelling lifestyle in a trailer in the distant or more recent past. The notion of "the travelling way" emerged as a central concept for this group. As one respondent stated, "(travelling), it's who we are". Another participant suggested the complexity as well as the enduring nature of this concept,

\section{"the travelling way ... I don't know what it is, but it never leaves you, never leaves you, no."}

Only one participant referred to the economic necessity of travelling,

\section{"Do you know why Travellers go away-to earn the money ... They can't stay in the same place ... It's a way of life." (M4)}

A focus on accommodation difficulties and the threat to the travelling lifestyle dominated many of the accounts given by our respondents. In describing their experiences of health and illness, this group referred directly to the effect of a travelling lifestyle, and often linked their health experiences to adversity experienced as a result of the limited accommodation options open to Gypsies and Travellers. These issues were often described in terms of perceived benefits and disadvantages of the travelling lifestyle.

Participants perceived many health-related benefits from "the travelling way" including freedom, choice and ability to live among extended family. Often the first benefit mentioned was "fresh air" associated with being outdoors, with strong regret expressed at the loss of this perceived benefit if a move to housing was deemed necessary.

\footnotetext{
"It's healthier for the kids travelling around because you're getting so much fresh air ..." (F20)
} 


\section{Box 1 The travelling way}

"I was happy if I was in a caravan ... freedom and all that ... you know, fresh air. I'm used to plenty of fresh air.." (M5)

"It used to be different because maybe you'd get two or three weeks in one place and you'd be with all different families and just nice ... I find it terrible, you miss out, and you miss it. You miss it for the children as well because the children really enjoy it, they like meeting all different people and living in all different places." (F13)

"So in other words you just couldn't stop anywhere, making you go out of the area. How can I go out of the area? If I go up that motorway and something happens to my wife, what am I going to do, stuck in the middle of the motorway? I don't know ... if you were ill you wouldn't want the police to come and say right, 'you've got to move out of your house,' That's what they're basically saying to you...you've got to get back in the motor and drive. Don't stay in our area because we're going to come and move you again." (M4)

"I blame moving there caused my health ... I mean it's a health hazard down there ... that's when my health started to go wrong. See I'm right against the motorway, then the tip and then the sewerage." (F2)

"The house is like toilets, baths, warmth, comfort that I didn' $t$ have in the caravan. I could handle the caravan before I was sick but there when I got unwell I couldn't, it was just getting too much for me ... because you'd have to move every day basically. Police would move you on ... so that weren't helping, not at all ... I wish I had my health back because I imagine I wouldn't be here. I don't know (sighs). It's just the way you're reared up isn't it." (Fl)

"We had a house in X, but we couldn't live in it ... the people on the housing estate, they were hurting the kids and one thing and another. They were big women fighting me 11-year-old daughter... and when the kids were going to school and getting bullied 'you're only Gypsies' and all the names under the sun." (F6)

Perceived benefits of a travelling lifestyle also included proximity to extended family members in an otherwise hostile "world". This was felt to be important, both psychologically and in terms of practical support and security. Travelling also allowed the possibility of moving away from potential trouble. Some participants described how they had returned to living in a trailer in order to escape hostility or victimisation from housed neighbours.

However, those trying to maintain a travelling lifestyle also described negative effects. Travelling was seen as potentially hazardous because of the diminishing choice of safe stopping places. Lack of basic amenities, such as running water, on unauthorised camping sites or poorly serviced transit sites, and difficulties in accessing services such as education and healthcare were other perceived disadvantages that sometimes precipitated a decision to seek a settled base. Health-related

'Each quote is attributed to the interviewee by their unique identification code. $M$ and $F$ stand for male and female. The number relates to the order in which each man or woman was interviewed. concerns about the conditions on official rented sites and the surrounding environment were also commonly reported. In addition to health risks from hazardous environments, psychological effects were also described, such as a feeling of "imprisonment" on a rented site related to the loss of freedom to travel. ${ }^{\mathrm{i}}$

For some participants, current poor health had precipitated a move into housing, although many felt coerced into this decision by additional circumstances, particularly the stress arising from being forcibly moved on from unofficial sites.

Elderly participants suggested that the wet and damp endured during years of living on the road in all weathers had had a detrimental effect on their health, with many blaming these conditions for their arthritis and chest complaints. Although health reasons were commonly cited for a move into housing, the psychological effect and "culture shock" of giving up a travelling lifestyle was perceived to counteract this benefit.

Among all age groups, a valued travelling lifestyle that was possible in the past was seen as increasingly denied and unattainable. Current adverse conditions, such as a lack of choice in accommodation and often intolerable conditions on existing sites, were described not only in terms of their direct effect but just as forcibly as an indication of the negative way in which society views Travellers and the travelling lifestyle. A perceived policy of social assimilation via accommodation and education was seen by many as a threat to their culture and identity as Gypsies and Travellers:

\begin{abstract}
"We haven't asked anybody to change their ways. Do you know what I mean? Why should they ask us to change ours? See what I'm saying. And that's what the government's trying to do. They're trying to make you change your ways."11 (M4)
\end{abstract}

\section{Low expectations of health}

An acceptance and expectation of ill health was pervasive, and some participants gave a long list of complaints suffered without considering themselves to be particularly unhealthy as a result. Many described their state of health, irrespective of its severity or extent, in terms of restrictions on their ability to perform daily tasks, and appeared to accept chronic ill health as long as day-today management of symptoms could be readily achieved.

The apparent acceptance of ill health and associated symptoms is consistent with an inverse relationship of access to healthcare in relation to need, although a lack of knowledge and understanding about the relevance of some symptoms was also evident. The inability to obtain relief for unmanageable symptoms was described, as was resignation and low expectations of improvement. Many Gypsies and Travellers who came to the presentations of the preliminary health survey findings ${ }^{5}$ were genuinely amazed that their overall state of health compared so unfavourably to other matched groups.

\section{Self-reliance and staying in control}

Stoicism and self-reliance were specific beliefs that were described as intrinsic for Gypsies and Travellers, arising from their experience of adversity and portrayed as necessary for survival. They were associated with the understatement of chronic ill health, commonly expressed as being tough and not admitting or succumbing to minor health complaints. Men in particular appeared to dismiss such complaints as insignificant.

Despite the fact that individuals took pride in self-reliance and in not "putting each other out" unless really necessary, most spoke of their responsibility to share care for their sick or elderly parents and extended family members. The idea of a relative going into a care home was particularly deplored. 


\section{Box 2 Low expectations of health}

"I don't feel top twenty like, but I feel fine." (Man with breathing, digestive and kidney problems, headaches and hypertension $-M 2$ )

"I don't think it's nothing too major ... I haven't had it forever, I think it's this last four or five months." (21-year-old man who coughed persistently through the interview-M4)

"I suffers with asthma and I had, must be getting on two years ago, I had about like, four or five mini strokes. Well I just took me tablets now for it [frozen shoulder] and that so I got tablets really to control that... arthritis, I got that and .... Like I can't um, like ... I can bounce about the place and just dust and do what I can reach and that, but there's like, clean windows, can't do anything like that ... so I still got this stuff ... when I starts walking and I finds I' $m$ getting a little pain or a bit breathless, I just takes that. So I'm very happy ..." (Woman with angina, frozen shoulder, arthritis, asthma and several "mini strokes" -F17)

"My health is perfect, lovely ... I only have a bad back all the time -that's since I was 15." (Woman with chronic back complaint, a history of severe allergies and skin complaintF5)

"I get tired, moody, restless but that's, I've been like that most of my life but it's got worse now. ... I don't know. But apart from that, not too bad really, but like I say I used to have stacks of energy. I'd clean and do and clean and do all the time but I can't be bothered now. It's. If I have a good day today... then tomorrow I just haven't got the strength to do it... I think just you feel run down all the time." (Woman with symptoms but apparent acceptance of depression-F9)

"I don't bother with it [offered investigation for cœliac disease]. No I don't bother with it that much, but I'd a very bad colour I'd be anaemic. It's partly over that. I has um iron injections most of the times. But then it drops back down again you see... there's not much they can do is there, just keep giving me the injections and bring it back up." (Woman with a history of childhood coeliac disease and severe anaemia-F13)

Self-reliance was also associated with a strong need to retain control, with high levels of stress arising from any inability to do so. One of the hardships often described by the respondents in this study was the lack of perceived control over their destiny, in the light of policies that were felt to restrict their self-determination and authorities that exclude them in various ways. The impotence felt by such unequal power relationships results in despair for some respondents, whereas others resisted domination and fought hard to achieve as much control as possible.

A strong sense was expressed of living within a society that is hostile, and has been for many generations, which seemed to reinforce the belief that they should be wary, and slow to invest their trust in outsiders, until convinced otherwise. A lack of

'Most Gypsies and Travellers are licensees on rented sites and can lose their licence without notice if they vacate their plot to travel for more than a specified limited period of weeks. Other restrictive conditions that gave the feeling of "imprisonment" were described.

\section{Box 3 Self-reliance and staying in control}

"You've got to be tough and get on with it." (M4)

"My family is there like, but you've got to help yourself."

"Somebody can be stressed out over something that can' $t$ be changed you know." (F11)

"You can't just sit and let yourself be depressed. You have to get on and do things. I think 'oh my children, they've lost their father and I have to carry on'." (F16)

"I don't want them to have a sick Mammy all my life, because I don't want to spoil it for them. So I just tell them 'Mammy doesn't feel too well, she has to lie down for a little while. I know it's lying to them, you know, but I don't want to ruin their childhood for them'." (F1)

"I said I felt great and happy and all that, you know, gave them a bluff." (M2)

"If you can't fight it yourself you're dead, you get worser, if you just think you haven't got it, just let go, try and control yourself, it's better... try and clean and leave it out of your head, because if you try and think, turn my depression off, you don't think I've got depression. I don't need to see the doctors, I don't have depression love, I say, I'm fine." (F20)

"My grandfather needed help for a bath and that. They wouldn't have home help. They looked after him. We don't believe in home help. We look after our own ourself." (F12)

"No [won't seek medical help] because I think I'll snap out of it won' $\mathrm{I}$ and just be calm and smoking." (F4)

"They [Traveller women in general] tend to make out, well I'm fed up but everything in the garden's lovely ... they don't confide, but people know anyway." (F9)

trust in others, with resulting low expectations, was sometimes manifested as stoicism and increased self-reliance.

\section{Fatalism, fear of death, avoidance}

While participants often expressed fatalism with regard to health problems, there was no evidence of any acceptance of life-threatening diagnoses or the experience of dying.

Fears around death and dying were strongly associated with a dread of a diagnosis of cancer, which was seen as an inevitable "death sentence", and this seemed to be confirmed by experience for most respondents. People therefore avoided any possibility of hearing that diagnosis. The fatalistic attitude that "nothing can be done" for cancer was frequently described, which in turn is likely to increase the chances of a terminal prognosis.

This finding, of an exceptionally strong and specific aversion to discussing cancer, was reinforced at one of our respondent validation sessions. There was a marked reduction of participation when cancer was mentioned; a lively and talkative group suddenly became uneasily silent, and showed little desire for discussing this aspect of the findings in a large forum.

Bereavement was also deeply feared and the intensity of grief related to bereavement described was severe and prolonged in all cases, with several stories of relatives "never getting over the 


\section{Box 4 Fatalism, fear of death, avoidance}

"If God borned you to have a short life, you had a short life, if he borned you to have a long life, you'd have a long lifenothing you could do to alter it." (F17)

"I started screaming, I said no, ... for the fright, for the shock of hearing it ... because I knew I was going to die right quick." (Woman, on being asked by the consultant examining her if there was a history in the family of bowel cancer-F8)

"I think cancer is one of the biggest fears of travelling people ... Because when that word is mentioned, cancer, it's like a tremble through the heart and I would talk to 5000 travelling people and [if] the first word is that, anything at all about that they just dies, they just falls to pieces." (Same woman (explaining her own reaction))

"A lot of Travellers blame [it on] if him or her didn't find out they had it [cancer], they'd live for another 30 years and all that ... a lot of people going for check ups have found out and when they've found out they've died straight away you know. But if they hadn't gone that's what they'd claim like ... He might live for another 10 or 20 years." (M2)

"No, I don't want to know, what I don't know can't hurt me." (Woman discussing health screening-F4)

death". The prolonged and extreme grief reactions described might appear surprising in the light of the close and supportive extended family that rally round when someone has died. However, there are several aspects to family involvement that appeared to give conflicting benefits. Close family members will ensure that the bereaved relatives are never left alone after the death. This would seem to be particularly important for Gypsy and Traveller families, as it would be so unusual in their experience to have ever been alone, and it adds further to the acute sense of loss experienced when a death occurs. Family members therefore experienced added grief on witnessing the grief of close relatives.

The grief of bereavement was perceived as a cause of illness and often as an indirect cause of death. Certain strategies people used to try to cope with bereavement and depression were recognised as an added cause of ill health, most notably, substance and alcohol misuse.

\section{DISCUSSION}

This study was limited by practical difficulties in accessing and interviewing members of this marginalised minority group. This particular subgroup was purposely selected as a group with health problems, and therefore the emergent themes from analysis of the individual interviews would not necessarily be applicable to all Gypsies and Travellers. However, these themes were presented to groups of Gypsies and Travellers, who endorsed them, recognised them as meaningful and amplified them. Consultations with stakeholders on the advisory group and within these forums add explanatory depth to the overall findings. The study findings are therefore likely to represent important health beliefs and attitudes among Gypsies and Travellers, to describe their experience of ill health, and to illuminate the high prevalence of health problems in this population, revealed in the health status survey. ${ }^{5}$ Ill health was directly attributed to social hardship and poor environmental conditions, which were widely reported by our sample, and also

\section{What is already known on this topic}

- Our survey of health status (see companion paper) showed an excess burden of ill health among Gypsies and Travellers in England.

- Ill health among Gypsies and Travellers exceeds that seen in other ethnic minorities and socially disadvantaged groups.

- Socioeconomic position and cultural beliefs influence health and health-seeking behaviour.

\section{What this study adds}

- Coherent cultural beliefs and attitudes underpin healthrelated behaviours among Gypsies and Travellers, combining low expectations, normalisation of ill health, stoicism, fatalism, ignorance and fear.

- Adverse social experiences and distressing perceptions of hostility and racism are also pervasively linked to ill health by respondents.

- Culturally appropriate and sensitive services are required to improve the health experience of Gypsies and Travellers.

to the distress that these cause in terms of their social meanings. For example, considerable distress was linked to the feeling of being "confined" and the erosion of freedom on a site, and isolation from extended family and the wider Gypsy Traveller culture arising from house dwelling. Great distress was reported from the perception that these hardships are imposed by a hostile and racist society.

There was an overriding perception of negative social attitudes and social exclusion as damaging to health, particularly mental health, but equal dismay at the prospect of social assimilation. These findings replicate those reported in an Irish study of

\section{Policy implications}

- We expect improvements in the policy response to the needs of this neglected minority in the light of these findings. Those responsible for public health policy and planning cannot assume that the health needs of Gypsies and Travellers are met by existing policy in relation to other ethnic minorities and socially disadvantaged groups.

- Strong ethnic identity and coherent cultural beliefs and attitudes underpin health-related behaviour in this group, and health experiences need to be understood in this context, alongside the specific effect of social and economic hardship and social exclusion.

- Health data are important in monitoring health and evaluating the effects of health interventions, and Gypsies and Travellers should be included in routine ethnic monitoring.

- The provision of effective healthcare and improvement of poor health in Gypsies and Travellers requires multiagency awareness and joint working to deal with the negative effects of both adverse social experiences and attitudinal barriers to health. 
Traveller health, the only other study that links epidemiological and qualitative findings. ${ }^{9}$ Although this qualitative study is unable to show causes of ill health among participants, the effect of both social adversity and personal health beliefs (such as "fatalism") on health outcomes is well evidenced-for example, in chronic diseases such as asthma, ${ }^{19}$ diabetes $^{20}$ and in mental illness. ${ }^{21}$ The link between health outcomes and the perception of Gypsies and Travellers that they are one of the most alienated groups in society is consistent with evidence from other studies.22 23

Both environmental and psychological factors are mirrored in the major themes arising from this study. The poor health reported from the health status survey ${ }^{5}$ was accompanied by low health expectations in this sample, but our participants did not see themselves as unusual in their experience of polymorbidity, and many described their experience of caring for family members with equally poor health.

The familiarity with ill health and premature death among family members in a group that has relatively little close contact with the rest of society may help to explain the apparent acceptance and normalisation of ill health and the neglect of certain aspects of self-care.

Self-reliance, stoicism, fatalistic health beliefs and avoidance of thinking about or discussing feared diseases such as cancer may also be linked to ignorance of the long-term implications of initial symptoms, making it easier to tolerate conditions if symptoms are manageable. The combination of a lack of knowledge, low expectations and fear reduces the likelihood of seeking early treatment, particularly for those conditions (eg, cancer and heart disease) where early detection has the greatest effect on prognosis.

Studies of health attitudes of other disadvantaged or disempowered groups, such as working class women and ethnic minorities, have also found a functional concept of health, fatalism and stoicism. ${ }^{24-26}$

However, these features do not account for all our findings, and others must be attributed to the particular culture of Gypsies and Travellers and associated adverse social experiences. The evidence in this study, showing the particular beliefs and circumstances that Gypsies and Travellers relate to their health experience, need to be taken account of and understood in the design of services aimed at improving their health.

\section{Authors' affiliations}

Patrice Van Cleemput, Glenys Parry, Jean Peters, Cindy Cooper, School of Health and Related Research, University of Sheffield, Sheffield, UK Kate Thomas, School of Healthcare, Baines Wing, University of Leeds, Leeds, UK

Funding: Funding was received from the Department of Health. The views expressed in this publication are those of the authors and not necessarily those of the Department of Health.

Competing interests: None.

\section{REFERENCES}

1 Liegeois JP. Gypsies and Travellers: socio-cultural data, socio-political data. Strasbourg: Council for Cultural Co-operation, 1987.

2 Crawley H. Moving forward: the provision of accommodation for Travellers and Gypsies. London: Institute of Public Policy Research, 2004.

3 Commission for Racial Equality. Common ground: equality, good race relations and sites for Gypsies and Travellers. London: CRE, 2006.

4 Anon in BBC News. CRE examines treatment of Gypsies, 17 Oct 2004. http:// news.bbc.co.uk/1/hi/england/3751214.stm
5 Parry G, Van Cleemput P, Peters J, et al. Health status of Gypsies and Travellers in England. J Epidemiol Community Health 2007;61:198-204.

6 Spencer L, Ritchie J Lewis J, et al. Quality in qualitative evaluation: a framework for assessing research evidence. London: National Centre for Social Research on behalf of the Cabinet Office, Government Chief Social Researcher's Office, 2003.

7 Ginnety P. The health of Travellers: based on a research study with Travellers in Belfast. Belfast: Eastern Health and Social Services Board, 1993.

8 Edwards DM, Watt RG. Diet and hygiene in the lives of Gypsy Travellers in Hertfordshire. Community Dent Health 1997;14:41-6.

9 Heron S, Barry J, Fitzgerald $M$, et al. The psychosocial health of Irish Traveller mothers. In: MacLachlan M, O'Connell M, eds. Cultivating pluralism:psychological, social, and cultural perspectives on a changing Ireland. Dublin: Oak Tree Press, 2000:93-116.

10 Streetly A. Health care for Travellers: one year's experience. BMJ 1987;294:492-4.

11 Crout L. Have healthcare will travel. Health Serv J 1988;98:48-9.

12 Batstone J. Meeting the health needs of Gypsies. Nurs Stand 1993;7:30-2.

13 Raper M. Travelling families in Northumberland. Health Visit 1986:59:345-6.

14 Windess B. One year working with the Travellers. Health Visit 1987:60:289-91.

15 Van Cleemput P. Health care needs of Travellers. Arch Dis Child 2000;82:32-7

16 Bodgewic SP. Participant observation. In: Crabtree B, Miller W, eds. Doing qualitative research; research methods in primary care. California: Sage Publications, 1992

17 Van Cleemput P, Parry G. Health status of Gypsy Travellers. J Public Health Med 2001;23:129-34

18 Ritchie J, Lewis J. Qualitative research practice: a guide for social science students and researchers. London: Sage, 2003.

19 Adams S, Pill R, Jones A. Medication, chronic illness \& identity: the perspective of people with asthma. Soc Sci Med 1997;45:189-201.

20 Greenhalgh T, Helman C, Chowdhury M. Heath beliefs and folk models of diabetes in British Bangladeshis: a qualitative study. BMJ 1998;316:978-83.

21 Bhui K, Fenton S, Grewal I, et al. Ethnic differences in the context and experience of psychiatric illness: a qualitative study. In: O'Connor W, Nazroo J, eds. Norwich: The Stationery Office, 2002.

22 Nazroo J. Patterns of and explanations for ethnic differences in health. In: Mason D, eds. Explaining ethnic differences:changing patterns of disadvantage in Britain. Bristol: The Policy Press, 2003:87-103.

23 Aspinall PJ, Jacobson B. Ethnic disparities in health and health care: a focused review of the evidence and selected examples of good practice. 17 Jan 2005. http://www. lho.org.uk/viewResource.aspx?id=8831.

24 Blaxter M, Paterson E. Mothers and daughters. London: Heinemann, 1982

25 Cornwell J. Hard earned lives: accounts of health and illness from East London. London: Tavistock, 1984.

26 Pill R, Stott N. Concepts of illness causation and responsibility: some preliminary data from a sample of working class mothers. Soc Sci Med 1982;16:52.

\section{APPENDIX A}

\section{SELF-REPORTED HEALTH PROBLEMS OF THE 27 INTERVIEWEES}

\section{Mental health problems}

Depression, anxiety, schizophrenia, "nervous disability" attributed to drug misuse

\section{Chronic physical problems}

Arthritis, hypertension, diabetes, angina, asthma, chronic back pain, chronic cough, anaemia and coeliac disease, diverticulitis, gastric ulcer, mild stroke

\section{Less well-defined problems}

Pregnancy with complications, "bladder problems", postoperative complications after bowel surgery, "kidney problems", chronic disability from connective tissue disease, investigations for cancer

\section{Problems associated with trauma}

Fractured vertebrae after a road traffic accident, laceration trauma to the leg

\section{Problems related to caring for close relatives with serious health problems}

Child with cerebral palsy, child with congenital syndrome 